Rev. Int. Contam. Ambie. 35 (Calidad de agua: salud, remediación y perspectiva) 93-101, 2019

DOI: 10.20937/RICA.2019.35.esp03.11

\title{
BIOCONCENTRACIÓN DE Pb, Cd Y As EN BIOMASA DE Eleocharis macrostachya (CYPERACEAE)
}

Bioconcentración de $\mathrm{Pb}, \mathrm{Cd}$ y As en biomasa de Eleocharis macrostachya (Cyperaceae)

\section{Brisa María ALDERETE-SUAREZ ${ }^{1}$, María Cecilia VALLES-ARAGÓN ${ }^{1 *}$, Sunem CANALES-REYES ${ }^{1}$, María del Rosario PERALTA-PÉREZ ${ }^{2}$ y Erasmo ORRANTIA-BORUNDA ${ }^{3}$}

${ }^{1}$ Facultad de Ciencias Agrotecnológicas, Universidad Autónoma de Chihuahua, Pascual Orozco y Universidad s/n, Campus I. C.P. 31200. Chihuahua, Chih., México

${ }^{2}$ Facultad de Ciencias Químicas, Universidad Autónoma de Chihuahua, Circuito Universitario, Campus II. C.P. 31124. Chihuahua, Chih., México

${ }^{3}$ Centro de Investigación en Materiales Avanzados S.C. Miguel de Cervantes 120, Complejo Industrial Chihuahua. C.P. 31136. Chihuahua, Chih. México

*Autora para correspondencia: valles.cecilia@gmail.com

(Recibido febrero 2018; aceptado octubre 2018)

Palabras clave: tratamiento agua, fitoextracción, traslocación, metales pesados

\section{RESUMEN}

Los crecientes problemas de contaminación del agua han creado espacios no aptos para habitar. La fitorremediación es una técnica que utiliza plantas para descontaminar agua, suelo y aire. Por ello, se evaluó la capacidad de Eleocharis macrostachya para remediar agua contaminada con plomo $(\mathrm{Pb})$, cadmio $(\mathrm{Cd})$ y arsénico $(\mathrm{As})$. Las plantas fueron recolectadas, se lavaron y trasplantaron en arena y se colocaron en macetas por grupos de tratamiento: T1 (testigo), T2 (As $2 \mathrm{mg} / \mathrm{L}, \mathrm{Cd} 10 \mathrm{mg} / \mathrm{L}, \mathrm{Pb} 20 \mathrm{mg} / \mathrm{L}$ ), T3 (As 4 mg/L, Cd 100 mg/L, Pb 200 mg/L) y T4 (As 8 mg/L, Cd 200 mg/L, Pb 400 mg/L). Las macetas fueron regadas por 210 días, al final del experimento se separaron suelo y planta para su digestión y posterior análisis con un espectrofotómetro de emisión óptica con plasma de acoplamiento inductivo (ICP-OES ). Se calcularon los factores de bioconcentración (FBC) y traslocación (FT) por metal y tratamiento. El FBC para los tres elementos fue mayor a 1 en todos los tratamientos. Para As el $\mathrm{FBC}$ varió de 5.4 a $4.0, \mathrm{Cd} 36.5$ a 19.6 y Pb 5.7 a 2.7. El FT para los tres elementos fue menor a 1 en T2 y mayor a 1 en T3 y T4. El intervalo de FT para As fue 0.6 a 4.7, Cd 0.5 a 2.6 y $\mathrm{Pb} 0.3$ a 3.2. E. macrostachya resultó ser fitoextractora, siendo capaz de acumular y traslocar $\mathrm{Pb}, \mathrm{Cd}$ y As; su capacidad se incrementó con el aumento de la concentración de los metales en el agua.

Key words: Water treatment, phytoextraction, traslocation, heavy metals

\begin{abstract}
The increasing problems of water pollution have created unhealthy spaces for inhabit. Phytoremediation is a technique that use plants to remediate water, soil and air. Therefore, the ability of Eleocharis macrostachya to remediate contaminated water with lead
\end{abstract}


$(\mathrm{Pb})$, cadmium $(\mathrm{Cd})$ and arsenic $(\mathrm{As})$ was analyzed. The plants were collected, washed and transplanted in sand and placed in pots by treatment groups: T1 (Control), T2 (As $2 \mathrm{mg} / \mathrm{L}, \mathrm{Cd} 10 \mathrm{mg} / \mathrm{L}, \mathrm{Pb} 20 \mathrm{mg} / \mathrm{L}$ ), T3 (As $4 \mathrm{mg} / \mathrm{L}, \mathrm{Cd} 100 \mathrm{mg} / \mathrm{L}, \mathrm{Pb} 200 \mathrm{mg} / \mathrm{L}$ ) and T4 (As $8 \mathrm{mg} / \mathrm{L}, \mathrm{Cd} 200 \mathrm{mg} / \mathrm{L}, \mathrm{Pb} 400 \mathrm{mg} / \mathrm{L}$ ). The pots were irrigated for 210 days, at the end of the experiment soil and plants were separated for digestion and subsequent analysis by inductively coupled plasma - optical emission spectrometry (ICP-OES). The bioconcentration (BCF) and translocation (TF) factors were calculated by kind of metal and treatment. The BCF for the three elements was greater than 1 in all the treatments. For As, BCF varied from 5.4 to 4.0, $\mathrm{Cd} 36.5$ to 19.6 and $\mathrm{Pb} 5.7$ to 2.7. The $\mathrm{TF}$ for the three elements was lower than 1 in T2, and greater than 1 in T3 and T4. The TF range for As was 0.6 to $4.7, \mathrm{Cd} 0.5$ to 2.6 and $\mathrm{Pb} 0.3$ to 3.2. E. macrostachya result to be phytoextractor, being able to accumulate and traslocate $\mathrm{Pb}, \mathrm{Cd}$ and $\mathrm{As}$; its capacity improved with the increase of metal concentrations in water.

\section{INTRODUCCIÓN}

Los metales pesados y metaloides son peligrosos para los seres vivos; su presencia en el ambiente puede causar contaminación del suelo y el agua, deterioro de la estructura del suelo, destrucción de paisajes y disminución de la biodiversidad (Bello et al. 2018). El agua es indispensable para la vida, sin embargo, debido a la contaminación por diversos medios su calidad y disponibilidad han disminuido severamente (Vera et al. 2016). Actualmente, se han detectado concentraciones tóxicas de metales pesados y metaloides en agua para usos agrícolas, en descargas de aguas residuales minero metalúrgicas, aguas residuales domésticas, e incluso en agua potable (Lyubenova et al. 2013).

Para descontaminar el agua existen diferentes técnicas. Una de ellas es la fitorremediación, que se define como el uso de plantas para absorber, acumular, eliminar, metabolizar, volatilizar o estabilizar contaminantes como metales pesados y metaloides, elementos radioactivos, compuestos orgánicos y compuestos derivados del petróleo en suelo, aire, agua o sedimentos (Delgadillo et al. 2011). Las plantas generalmente toleran contaminantes sin afectar la tierra y pueden mejorar la fertilidad del suelo introduciendo materia orgánica (Mench et al. 2009). Éstas tienen diversos mecanismos celulares que puede estar involucrados en la desintoxicación de metales pesados y metaloides y confieren tolerancia al estrés causado por dichos elementos. Cuando las toxinas están presentes en concentraciones elevadas, las células de las plantas activan una red de almacenamiento y procesos de desintoxicación, como la quelación de iones metálicos en el citosol, seguida del envío y captura en la vacuola (Yadav et al. 2018).

Dentro de los tipos de fitorremediación para medios acuosos contaminados con metales pesados y metaloides, están la fitoextracción y la fitoestabilización (Bayón 2015). La fitoextracción o hiperacumulación combina los mecanismos de adsorción, transporte y traslocación de metales pesados y metaloides a partes cosechables de la planta (Barzanti et al. 2011). La fitoestabilización es utilizada para reducir la movilidad y la biodisponibilidad de los contaminantes en el ambiente, evitando así la migración hacia el agua subterránea o introducción en la cadena trófica (Ali et al. 2013). Es decir, las plantas pueden adoptar distintas estrategias para contrarrestar la toxicidad de metales en su entorno. Unas basan su resistencia en una eficiente exclusión del metal, restringiendo su transporte a la parte aérea. Otras acumulan el metal en la parte aérea, en una forma no tóxica para sí mismas (Núñez et al. 2007). Sin embargo, se destaca que la transferencia de los metales pesados y metaloides del suelo a las plantas depende de la especiación química en la que se encuentren, tipo de suelo, $\mathrm{pH}$ del suelo, materia orgánica, capacidad de intercambio catiónico, contenido de arcilla y potencial óxido reducción (Ali et al. 2013). Asimismo, de acuerdo con Kersten et al. (2017), el uso de material vegetal nativo es recomendable para un óptimo proceso de fitorremediación.

Eleocharis macrostachya es una planta hidrófita, emergente, perenne, nativa de la región norte de México, de la familia de los juncos (Cyperaceae). Presenta hasta $60 \mathrm{~cm}$ de altura, con rizomas color café rojizos, de $30 \mathrm{~cm}$ de largo y hasta $2.5 \mathrm{~mm}$ de diámetro, sobrevive a condiciones climatológicas extremas, tanto de verano hasta $45{ }^{\circ} \mathrm{C}$ como de invierno soportando hasta $-8{ }^{\circ} \mathrm{C}$ (Núñez et al. 2007). Debido al clima extremoso en el territorio del estado de Chihuahua $\left(-15^{\circ} \mathrm{C}\right.$ a $48{ }^{\circ} \mathrm{C}$, CONABIO 2014), no resulta fácil elegir una planta para su uso en fitorremediación, por lo que se ha estado evaluando 
el potencial de E. macrostachya. En investigaciones anteriores, esta planta resultó exitosa para la remoción de As en agua de rechazo del tratamiento de osmosis inversa, probada en maceta con concentraciones de As en agua de hasta $5 \mathrm{mg} / \mathrm{L}$ y en humedal de hasta $0.5 \mathrm{mg} / \mathrm{L}$ (Olmos-Márquez et al. 2012); asimismo, fue propuesta para el tratamiento de agua para uso y consumo humano con concentraciones de $0.1 \mathrm{mg} / \mathrm{L}$ (Valles-Aragón y Alarcón-Herrera 2014). Por ello, en esta investigación se estableció analizar la capacidad de E. macrostachya para remediar aguas contaminadas con $\mathrm{Pb}, \mathrm{Cd}$ y As y evaluar el potencial de la planta en su utilización a nivel prototipo para el tratamiento de aguas residuales industriales.

\section{MATERIALES Y MÉTODOS}

\section{Colecta y preparación de plantas}

Se recolectaron plantas de la especie Eleocharis macrostachya a $53 \mathrm{~km}$ de la ciudad de Chihuahua, en escorrentías de aguas termales de la localidad de San Diego de Alcalá $\left(28^{\circ} 35^{\prime} 05^{\prime \prime} \mathrm{N}, 105^{\circ} 34^{\prime} 22\right.$ ” O), a finales de la temporada de verano. Las plantas, en floración, fueron transportadas al laboratorio en bandejas de plástico con sustrato y agua del sitio. En el laboratorio fueron separados brotes del rizoma en tamaños proporcionalmente iguales, éstos se limpiaron y trasplantaron a bolsas de plástico negro $(8 \times 15 \mathrm{~cm})$. Se colocó un brote en cada maceta y como medio de soporte se utilizó arena limosa (caracterizada previamente) a una altura de $10 \mathrm{~cm}$ (2.5 kg por bolsa). Previo al experimento, se mantuvieron en condiciones de saturación con agua del grifo, para estabilizar las plantas por dos meses dentro de invernadero y permitir el desarrollo de nuevos rizomas.

\section{Caracterización de agua y suelo}

Se analizaron parámetros físicos y químicos del agua de grifo y la arena limosa (suelo) que se utilizaron como agua de riego y medio de soporte para plantas en el experimento. En el agua se determinaron el potencial óxido reducción (ORP) y el potencial hidrógeno $(\mathrm{pH})$ con un potenciómetro Aqua; conductividad eléctrica (CE) y sólidos disueltos totales (SDT) con conductímetro Hanna. En el suelo se determinaron textura por el método de Bouyoucos, contenido de materia orgánica (MO) por el método Walkey y Black, $\mathrm{pH}$ con el potenciómetro Aqua, CE con conductímetro Hanna y capacidad de intercambio catiónico (CIC) con cálculo indirecto de textura y MO.

\section{Configuración del experimento}

Se establecieron cuatro grupos de tratamiento formados por cinco macetas cada uno. El primer grupo (T1) fue el asignado como testigo; éste se regó con agua de grifo (previamente caracterizada). Para los tres grupos restantes se consideraron los límites máximos permitidos en la NOM-002-SEMARNAT-1996 para la descarga de aguas residuales (As $1 \mathrm{mg} / \mathrm{L}, \mathrm{Pb}$ 2 mg/L y Cd 1 mg/L) (SEMARNAT 1996). El segundo grupo (T2) se regó con agua sintética preparada con As $(2 \mathrm{mg} / \mathrm{L}$, dos veces más que el límite establecido), $\mathrm{Pb}$ y Cd (20 y $10 \mathrm{mg} / \mathrm{L}$, respectivamente, diez veces más que el límite establecido). El tercer grupo (T3) se regó con agua sintética preparada con As (4 mg/L, cuatro veces más que el límite establecido), $\mathrm{Pb}$ y $\mathrm{Cd}$ (200 y $100 \mathrm{mg} / \mathrm{L}$, respectivamente, 100 veces más que el límite establecido). Por último, el cuarto grupo (T4) se regó con agua sintética preparada con As ( $8 \mathrm{mg} / \mathrm{L}$, ocho veces más que el límite establecido), $\mathrm{Pb}$ y Cd (400 y $200 \mathrm{mg} / \mathrm{L}$, respectivamente, 200 veces más que el límite establecido) (Fig. 1). El agua sintética fue preparada con agua de grifo arsenito de sodio $\left(\mathrm{AsNaO}_{2}\right)$, cloruro de cadmio $\left(\mathrm{CdCl}_{2}\right)$ y cloruro de plomo $\left(\mathrm{PbCl}_{2}\right)$ para los tratamientos T2, T3 y T4.

Las plantas fueron regadas con el agua de grifo (T1) y agua sintética preparada (T2 - T4) cada tercer día, por un periodo de 210 días. Al final del experimento se retiraron las plantas del suelo, para su preparación y análisis.

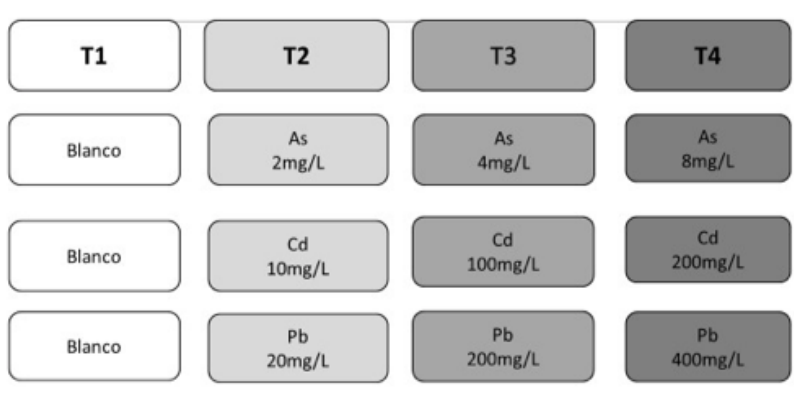

Fig. 1. Diseño de experimentos: concentraciones de As, Cd y $\mathrm{Pb}$ por tratamientos

\section{Análisis de plantas y suelo}

Las plantas se lavaron cuidadosamente con agua destilada para retirar el exceso de sedimento y suelo adherido a la planta. Después se separaron en sus diferentes partes (raíz y parte aérea). Cada parte se pesó en húmedo, posteriormente se secaron a temperatura ambiente $\left(30 \pm 5^{\circ} \mathrm{C}\right)$ y se molieron. Las 
plantas fueron digeridas en un microondas Mars6 por el método EPA 3052. Para la determinación de la concentración de $\mathrm{As}, \mathrm{Cd}$ y $\mathrm{Pb}$ en cada parte de la planta, las muestras en forma líquida fueron analizadas por plasma de acoplamiento inductivo junto a un espectrofotómetro de emisión óptica (ICP-OES) en un equipo Thermo Scientific modelo iCAP 6500.

El suelo de cada maceta y tratamiento se pesó y secó a temperatura ambiente $\left(30 \pm 5^{\circ} \mathrm{C}\right)$. La digestión de suelo se realizó acorde al método EPA 3051 en microondas marca Mars6, posteriormente se determinó la concentración de $\mathrm{As}, \mathrm{Cd}$ y $\mathrm{Pb}$ por ICP-OES. Para el análisis de las muestras tanto de suelo como de planta, se utilizaron blancos y réplicas.

\section{Cálculo de factores de traslocación y bioconcen- tración}

Se calcularon los factores de traslocación (FT) y bioconcentración (FBC) de la especie E. macrostachya para $\mathrm{As}, \mathrm{Cd}$ y $\mathrm{Pb}$ para determinar su eficiencia en el proceso de fitorremediación.

El factor de bioconcentración es la relación entre la acumulación del metal en el agua y en la biomasa de la planta (Yadav et al. 2018).

$F B C=\frac{\text { C tejido cosechado }}{\text { Cagua }}$

$\mathrm{FBC}=$ Factor de bioconcentración

$\mathrm{C}$ tejido cosechado $=$ Concentración en tejido cosechado

$\mathrm{C}$ agua $=$ Concentración en agua
Valores del FBC mayores a 1 indican que la planta tiene la capacidad de bioacumular el metal o los metaloides en su biomasa (Ali et al. 2013).

El FT es el cociente entre la concentración del metal en los órganos aéreos y la raíz de la planta (Bello et al. 2018).

$F T=\frac{\text { Caérea }}{\text { Craiz }}$

$\mathrm{FT}=$ Factor de traslocación

$\mathrm{C}$ aérea $=$ Concentración en tejido cosechado, parte aérea

$C$ raíz $=$ Concentración en tejido cosechado de raíz

Factores de traslocación mayores a 1 sugieren gran capacidad para transportar metales desde las raíces hasta los vástagos (Ali et al. 2013).

\section{Análisis estadístico}

Se realizó un análisis estadístico por medio del programa SPSS Statistics 20 para la comparación de la biomasa seca de las plantas y para la comparación y análisis de los FT y FBC.

\section{RESULTADOS Y DISCUSIÓN}

\section{Parámetros físicos y químicos de agua y suelo}

En el Cuadro I se presentan las magnitudes y clasificaciones de los parámetros físicos y químicos analizados en el agua de grifo y suelo (arena) que se utilizaron en el experimento. El suelo utilizado

CUADRO I. PARÁMETROS FÍSICOS Y QUÍMICOS DE AGUA Y SUELO

\begin{tabular}{|c|c|c|c|c|c|c|}
\hline Parámetro & Matriz & Unidad & Valor & Clasificación & Norma & Método \\
\hline Textura & Suelo & $\%$ & 91 & Arena & (RECNAT 2000) & Bouyoucos \\
\hline$\overline{\mathrm{MO}}$ & Suelo & $\%$ & 1.3 & Muy bajo & (RECNAT 2000) & Walkey y Black \\
\hline$\overline{\mathrm{pH}}$ & Suelo & Adimensional & 8.2 & Medianamente alcalino & (RECNAT 2000) & Potenciómetro Aqua \\
\hline$\overline{\mathrm{CE}}$ & Suelo & $\mathrm{dS} / \mathrm{m}$ & 1.4 & Ligeramente salino & (RECNAT 2000) & $\begin{array}{l}\text { Potenciómetro portátil } \\
\text { Hanna }\end{array}$ \\
\hline$\overline{\mathrm{CIC}}$ & Suelo & $\mathrm{Cmol}+/ \mathrm{kg}$ & 3.5 & $\begin{array}{l}\text { Baja fertilidad debido a su } \\
\text { baja reserva nutrimental }\end{array}$ & (RECNAT 2000) & $\begin{array}{l}\text { Cálculo indirecto con } \\
\text { MO y Textura }\end{array}$ \\
\hline$\overline{\mathrm{ORP}}$ & Agua & $\mathrm{mV}$ & 51.4 & Condiciones reductoras & No se regula & Potenciómetro Aqua \\
\hline$\overline{\mathrm{pH}}$ & Agua & Adimensional & 7.1 & $\begin{array}{l}\text { Neutro, dentro de los límites } \\
\text { reglamentados para uso } \\
\text { agrícola }\end{array}$ & (CONAGUA 2017) & Potenciómetro Aqua \\
\hline$\overline{\mathrm{CE}}$ & Agua & $\mathrm{dS} / \mathrm{m}$ & 0.7 & $\begin{array}{l}\text { No salina, no se restringe su } \\
\text { uso agrícola }\end{array}$ & (Ayers y Westcot 1994) & $\begin{array}{l}\text { Conductivímetro portátil } \\
\text { marca Hanna }\end{array}$ \\
\hline$\overline{\mathrm{SDT}}$ & Agua & ppt & 0.3 & $\begin{array}{l}\text { Dentro de los límites } \\
\text { reglamentados para uso } \\
\text { agrícola }\end{array}$ & (Ayers y Westcot 1994) & $\begin{array}{l}\text { Conductivímetro portátil } \\
\text { marca Hanna }\end{array}$ \\
\hline
\end{tabular}


fue arena $(91 \%)$, con bajo contenido de MO (1.3\%), $\mathrm{pH}$ medianamente alcalino (8.2) y ligeramente salino $(1.4 \mathrm{dS} / \mathrm{m})$. El agua se clasificó como no salina $(0.7 \mathrm{dS} / \mathrm{m})$, de $\mathrm{pH}$ neutro (7.1).

Se observó que las características físicas y químicas del suelo utilizado no eran las óptimas para incentivar la acumulación de metales pesados, debido a que la biodisponibilidad y absorción de éstos se incrementa a pH ácidos (Sheoran et al. 2016). Asimismo, está definida una mayor movilidad de metales pesados y metaloides a altos contenidos de materia orgánica en el suelo. Aunado a todo lo anterior, el suelo ligeramente salino es un factor que expone a la planta a una condición de estrés (Yadav et al. 2018). Sin embargo, la textura del suelo (arena) beneficia la disponibilidad de los metales pesados y metaloides para las plantas (Sheoran et al. 2016).

\section{Biomasa seca}

En la figura 2 se muestra la media del peso seco de la parte aérea de las plantas por tratamiento, donde se observó una mayor biomasa en T3. El valor medio de la biomasa seca del tratamiento más contaminado (T4) fue igual al valor medio del testigo (T1), lo que representó que los metales no tuvieran un impacto en el desarrollo de la biomasa (Fig. 2).

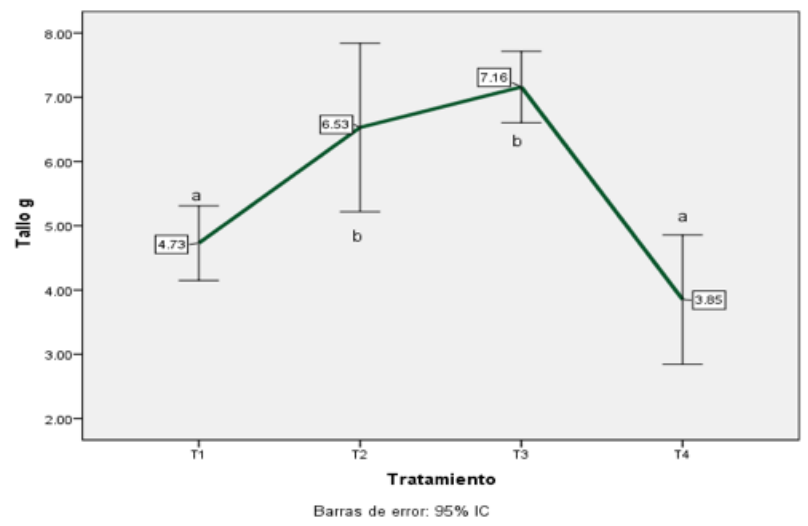

Fig. 2. Media de peso seco de parte aérea por tratamiento

En la figura 3 se observa que los pesos de raíz fueron mayores a la parte aérea, debido a que el rizoma que desarrolla E. macrostachya es leñoso y de gran tamaño. Se observó que en los pesos secos de raíz, T3 generó mayor cantidad de biomasa que los otros tratamientos. Al igual que en la parte aérea, las medias de biomasa de raíz en el testigo (T1) y el tratamiento más contaminado (T4) fueron similares (Fig. 3).

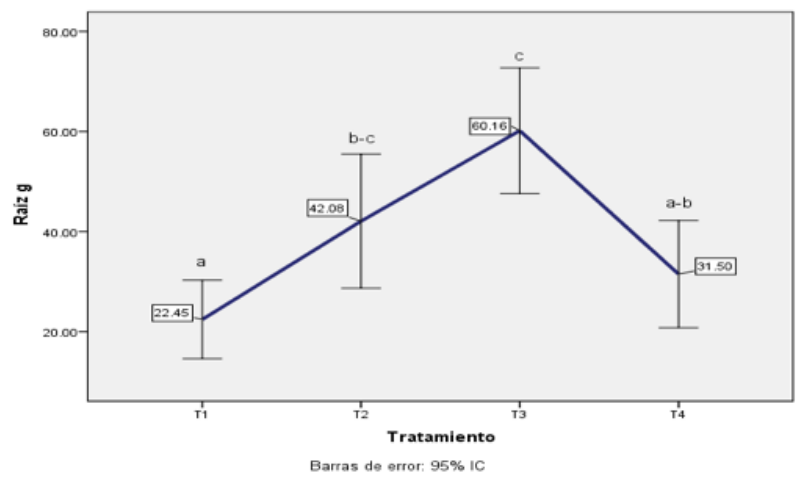

Fig. 3. Media de peso seco de raíz por tratamiento

Contrario a este resultado, Kersten et al. (2017) obtuvieron una diferencia significativa entre la biomasa del testigo de tres diferentes especies de sauce y los tratamientos con $\mathrm{Cd}(0.011$ y $0.3 \mathrm{mg} / \mathrm{L})$ y $\mathrm{Pb}(0.015$ y $0.145 \mathrm{mg} / \mathrm{L})$, donde se presentó una disminución en el crecimiento de la biomasa en las especies tratadas.

Asimismo, varios autores (Ali et al. 2013, Sheoran et al. 2016, Yadav et al. 2018) concuerdan en que la presencia de metales pesados y metaloides en general reduce el crecimiento de las plantas, incluso mencionan la necesidad de adicionar promotores de crecimiento. No obstante, también está demostrado que Thlaspi caerulescens presenta un óptimo crecimiento con la adición de Cd. En consecuencia, se estima que los efectos promotores del crecimiento por la presencia de $\mathrm{Cd}$ pueden ser un efecto indirecto de la interferencia a la disponibilidad de elementos nutricionales "reales" (Verbruggen et al. 2009).

\section{Análisis y determinación de metales en planta y suelo \\ Arsénico}

En raíz se observó presencia de As en T1 (testigo); además, en T2, T3 y T4 se determinó una mayor absorción conforme se incrementó la concentración del metaloide por tratamiento, variando de 42.8, 47.7 a $49.0 \mathrm{mg} / \mathrm{kg}$, respectivamente. La parte aérea absorbió mayor cantidad de As en T3 $(44.9 \mathrm{mg} / \mathrm{kg})$ y menor en T4 $(7.2 \mathrm{mg} / \mathrm{kg})$. En el suelo la concentración de As aumentó con el incremento de la concentración del metaloide en agua por tratamiento $(4.0$ - T1, 7.7 - T2, 16.4 - T3 y 39.3 - T4 mg/kg) (Fig. 4).

\section{Plomo}

$\mathrm{La}$ absorción de $\mathrm{Pb}$ en la raíz fue mayor en $\mathrm{T} 3$ (1164.6 mg/kg) y sólo un poco más baja en T4 $(801.8 \mathrm{mg} / \mathrm{kg})$. En la parte aérea la concentración de $\mathrm{Pb}$ fue mayor en T3 $(1549.7 \mathrm{mg} / \mathrm{kg})$ que en T4 


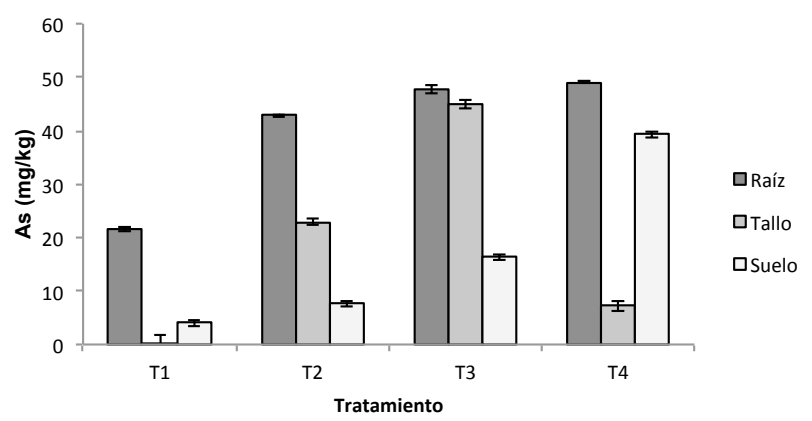

Fig. 4. Absorción de arsénico $(\mathrm{mg} / \mathrm{kg})$ en raíz, tallo y suelo, por tratamiento

(74.0 mg/kg). T2 y T4 mostraron niveles de absorción del metal semejantes $(75.4$ y $74.0 \mathrm{mg} / \mathrm{kg}$ ). En el suelo en T4 se observó la mayor concentración de $\mathrm{Pb}(1205.7 \mathrm{mg} / \mathrm{kg})$, seguido de T3 (461.8 mg/kg). Además, $\mathrm{T} 1$ mostró una mayor concentración de $\mathrm{Pb}$ que T2 (54.2 y $32.2 \mathrm{mg} / \mathrm{kg}$, respectivamente) (Fig. 5).



Fig. 5. Absorción de plomo $(\mathrm{mg} / \mathrm{kg})$ en raíz, tallo y suelo, por tratamiento

\section{Cadmio}

Se evidenció mayor absorción de Cd en la raíz conforme la concentración del metal en agua de los tratamientos aumentó; las medias de concentración de Cd en raíz variaron de $1.7,68.8,526.0$ hasta 2469.0 $\mathrm{mg} / \mathrm{kg}$, para T1, T2, T3, y T4, respectivamente. El Cd en la parte aérea fue mayor en T4 $(1884.7 \mathrm{mg} / \mathrm{kg})$ seguido de T3 $(933.7 \mathrm{mg} / \mathrm{kg})$, en T1 fue casi nula la absorción de $\mathrm{Cd}(0.5 \mathrm{mg} / \mathrm{kg})$. La concentración de Cd en el suelo fue mayor en T4 (493.3 mg/kg), el T2 mostró muy poca presencia de $\mathrm{Cd}$ en el suelo $(2.9 \mathrm{mg} / \mathrm{kg})$. En T1 se detectó una presencia casi nula del elemento $(0.3 \mathrm{mg} / \mathrm{kg})$ (Fig. 6).

Se considera que la presencia de $\mathrm{As}$ y $\mathrm{Pb}$ en la raíz de E. macrostachya en T1 pudo deberse a la presencia natural de este elemento en el sedimento de la zona en la que fueron recolectadas las plantas (Moreno-López et al. 2011).

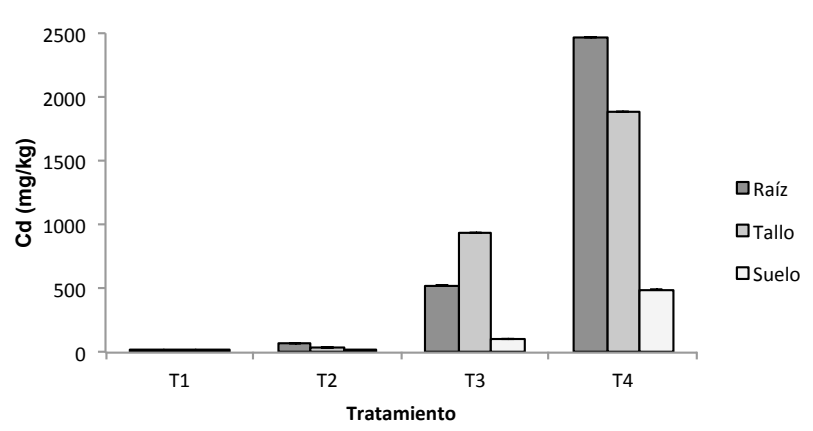

Fig. 6. Absorción de cadmio $(\mathrm{mg} / \mathrm{kg})$ en raíz por tratamiento

\section{Factores de bioconcentración y traslocación}

Para el cálculo de los factores de bioconcentración (FBC) y traslocación (FT) en los tratamientos T2, T3, $\mathrm{T} 4$, se consideró la concentración media de As, Cd y $\mathrm{Pb}$ presentes en la raíz y el tallo en el tratamiento T1 (testigo del experimento) como la concentración inicial de los metales y el metaloide en las plantas.

El FBC-As fue mayor a 1 en los tres tratamientos (T2 5.4, T3 4.2, T4 4.0) (Fig. 7). El FBC-Cd fue mayor en T2, sin embargo, en los tres tratamientos fue mayor a 1 (T2 36.5, T3 23.5, T4 19.6) (Fig. 8).



Fig. 7. Factor de bioconcentración de arsénico por tratamiento

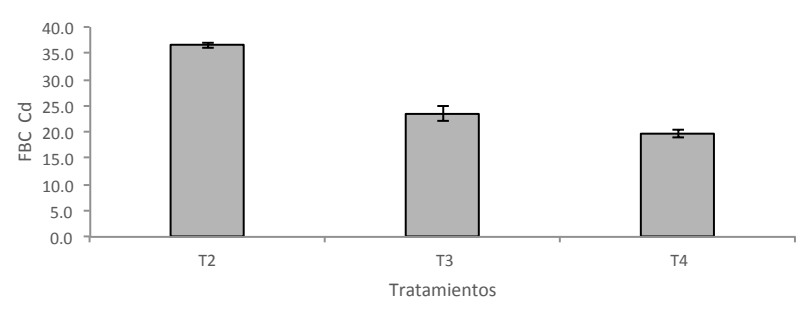

Fig. 8. Factor de bioconcentración de cadmio por tratamiento

E1 FBC-Pb fue mayor en T2, sin embargo, en los tres tratamientos fue mayor a $1(\mathrm{~T} 25.7, \mathrm{~T} 32.9, \mathrm{~T} 4$ 2.7) (Fig. 9).

Eleocharis macrostachya se clasifica entonces como una planta acumuladora, debido a que en todos los tratamientos se obtuvieron magnitudes mayores a 1, destacando que el FBC es una forma confiable 


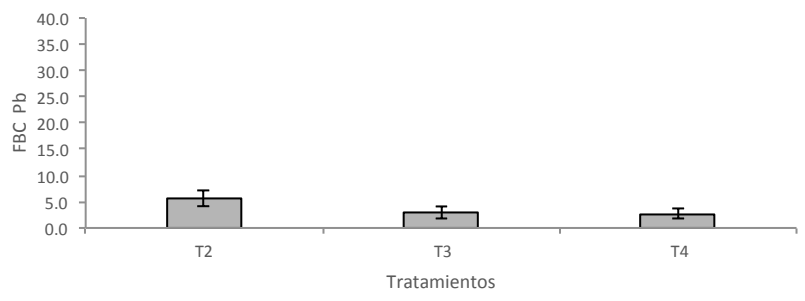

Fig. 9. Factor de bioconcentración de plomo por tratamiento

para determinar la biodisponibilidad de los metales y metaloides hacia las plantas (Ali et al. 2013).

El FT-As en T2 fue menor a 1 (0.6); en el caso de T3 y T4 fue mayor a 1 (T3 2.1 y T4 4.7), lo que representó que el As se traslocó a la parte aérea de la planta (Fig. 10). Otros autores con plantas diferentes demostraron comportamiento semejante. Luna Martínez (2011) germinó Lolium perenne (zacate ballico) en macetas utilizando suelo contaminado con As $(100 \mathrm{mg} / \mathrm{kg})$, donde determinó mayor acumulación del metaloide en tallo $(82.5 \mathrm{mg} / \mathrm{kg})$ que en raíz $(66.0$ $\mathrm{mg} / \mathrm{kg}$ ). Núñez et al. (2007) utilizaron de igual manera E. macrostachya en macetas, adicionaron As en soluciones de: $0,3,6$ y $9 \mathrm{mg} / \mathrm{L}$ y establecieron que la mayor concentración del metaloide fue en la parte aérea (95\% de retención). Valles-Aragón (2014) por otra parte, probó E. macrostachya en sistema de humedales construidos en flujo continuo con una concentración de As de $0.1 \mathrm{mg} / \mathrm{L}$ y determinó una mayor retención de As en la raíz.

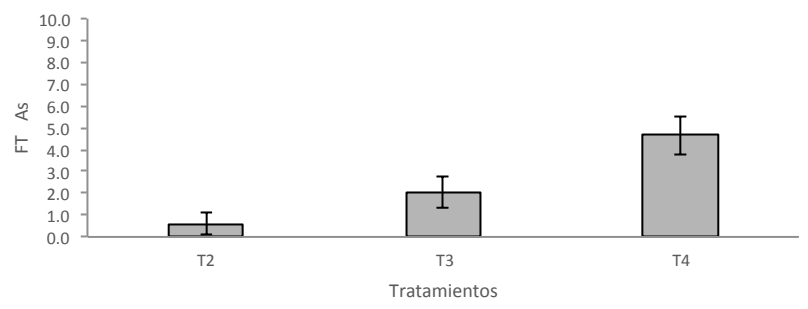

Fig. 10. Factor de traslocación de arsénico por tratamiento

El FT-Cd fue menor a 1 en T2 (0.5); en T3 y T4 fue mayor a 1 (1.8 y 2.6, respectivamente) lo que representó que en estos tratamientos la mayor concentración de $\mathrm{Cd}$ se quedó en la parte aérea (Fig. 11). Este resultado es semejante a los que obtuvo Hernández (2009), quien encontró en jales mineros mayor acumulación de $\mathrm{Cd}$ en la parte aérea de la planta $A$. Fistulosus que en las raíces. Asimismo, Kersten et al. (2017), analizaron tres especies de sauce con diferentes tratamientos de $\mathrm{Cd}(0.011 \mathrm{y}$ $0.3 \mathrm{mg} / \mathrm{L})$ y $\mathrm{Pb}(0.015$ y $0.145 \mathrm{mg} / \mathrm{L})$, y determinaron que las tres especies acumularon más $\mathrm{Cd}$ y $\mathrm{Pb}$ en el tallo. La investigación de Luna Martínez (2011) con Lolium perenne y Bouteloua gracilis (zacate navajillo) en macetas con suelo contaminado con $1460 \mathrm{mg} / \mathrm{kg}$ de $\mathrm{Cd}$, demostró que en ambas plantas hubo mayor retención del metal en las hojas.

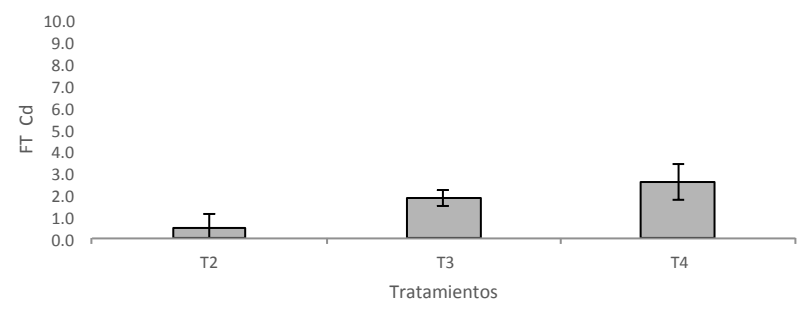

Fig. 11. Factor de traslocación de cadmio por tratamiento

El FT-Pb fue menor a 1 en el T2 (0.3); en los tratamientos T3 y T4 fue mayor a $1(1.5$ y 3.2 , respectivamente), lo que representó que en estos tratamientos la mayor concentración de $\mathrm{Pb}$ se quedara en la parte aérea (Fig. 12). Caso contrario, Ortiz et al. (2009) utilizaron Amaranthus hybridus L. (quelite), el cual germinaron y trasplantaron a macetas a las que se les adicionaron $300 \mathrm{mg} / \mathrm{kg}$ de $\mathrm{Pb}$, además de añadir micorrizas (Entrophospora columbiana, Glomus intraradices, G. etunicatum y G. clarum); los resultados de estos autores mostraron que la raíz de la planta acumuló mayor cantidad de $\mathrm{Pb}$ que otras partes.

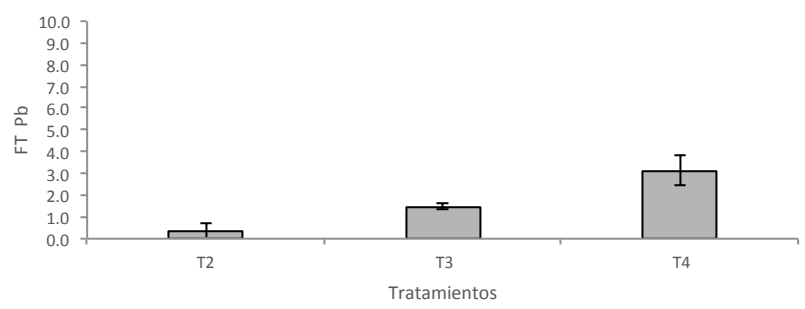

Fig. 12. Factor de traslocación de plomo por tratamiento

Los factores de bioconcentración y traslocación se clasificaron de forma semejante para cada metal en cada tratamiento. Es decir, el tratamiento donde el FBC fue mayor para todos los metales fue el T2, y el menor fue el T4. Lo anterior significa que la capacidad de bioconcentración de $\mathrm{As}, \mathrm{Pb}$ y Cd por la planta disminuye con el incremento de la concentración de los metales en el agua de riego (FBC T2 $>$ T3 $>$ T4). Sin embargo, en todos los tratamientos se tuvo una respuesta de bioconcentración alta. En el caso del factor de traslocación, este fue mayor 
que 1 en todos los metales en los tratamientos T3 y T4. Lo que representa que E. macrostachya a bajas concentraciones retiene los metales a nivel de raíz, posteriormente empieza a traslocarlos a la parte aérea.

Después de la entrada a las raíces, los iones de metales y el metaloide se almacenaron en los vasos del xilema, donde se depositaron principalmente en vacuolas (Ali et al. 2013). A bajas concentraciones, los metales y el metaloide se conservaron en la raíz de las plantas; al incrementar las concentraciones, éstos comenzaron a transportarse a la parte aérea por la corriente de transpiración a través del xilema (Yadav et al. 2018). E. macrostachya se consideró como planta acumuladora, pues presentó FBC mayores a 1. Las plantas con FBC y FT mayores a 1 pueden ser consideradas como fitoextractoras (Bello et al. 2018).

\section{CONCLUSIONES}

La planta Eleocharis macrostachya tiene potencial para fitorremediar aguas contaminadas, pues la biomasa de las plantas no se afectó por la presencia de los metales y el metaloide en el agua de riego. Conjuntamente demostró capacidad de acumular As, $\mathrm{Pb}$ y en mayor medida $\mathrm{Cd}$. Conservó el $\mathrm{As}, \mathrm{Pb}$ y $\mathrm{Cd}$ en la raíz a bajas concentraciones, y lo traslocó a la parte aérea con el aumento de la concentración de los metales y el metaloide en agua, comportamiento que fue semejante para los tres elementos.

El tratamiento testigo exhibió presencia de los elementos analizados sobre todo de $\mathrm{Pb}$ y As, lo cual puedo deberse a que dichos elementos se encuentran naturalmente en el medio del que fueron recolectadas las plantas.

La fitorremediación con Eleocharis macrostachya como técnica para la remediación de agua contaminada con $\mathrm{As}, \mathrm{Pb}$ y $\mathrm{Cd}$ puede ser efectiva ya que no causan alteraciones al ambiente por ser plantas que se encuentran naturalmente en el ecosistema chihuahuense. Sin embargo, debe de ser evaluada la viabilidad del uso de esta planta en un sistema de humedales construidos con agua contaminada con $\mathrm{As}, \mathrm{Pb}$ y $\mathrm{Cd}$.

\section{AGRADECIMIENTOS}

Al Centro de Investigación en Materiales Avanzados (CIMAV), por el apoyo para el análisis de muestras, específicamente a los ingenieros Silvia Miranda y Alejandro Benavides. Asimismo, al Programa para el Desarrollo Profesional Docente para el Tipo
Superior (PRODEP) por el apoyo económico dentro del proyecto con folio UACH-PTC-328.

\section{REFERENCIAS}

Ali H., Khan E. y Anwar M. (2013). Phytoremediation of heavy metals- Concepts and application. Chemosphere 7 (91), 869-881.

DOI: 10.1016/j.chemosphere.2013.01.075.

Ayers R.S. y Westcot D.W. (1994). Water quality for agriculture. Food and Agriculture Organization of the United Nations, Rome, Italy, 97 pp.

Barzanti R., Colzi I., Arnetoli M., Gallo A., Pignattelli S., Gabbrielli R., y Gonnelli C. (2011). Cadmium phytoextraction potential of different Alyssum species. J Hazard Mater. 196, 66-72. DOI:10.1016/j.jhazmat.2011.08.075.

Bayón S. (2015). Aplicación de la fitorremediación a suelos contaminados con metales pesados. Universidad Complutense. Departamento de Edafología, Madrid, España, 23 pp.

Bello A.O., Tawabini B.S., Khalil A.B., Boland C.R., y Saleh T.A. (2018). Phytoremediation of cadmium-, lead-and nickel-contaminated water by Phragmites australis in hydroponic systems. Ecol Eng. 120, 126133. DOI: 10.1016/j.ecoleng.2018.05.035.

CONABIO. (2014). La biodiversidad en Chihuahua: Estudio de Estado. Comisión Nacional para el Conocimiento y Uso de la Biodiversidad, México, 560 pp.

CONAGUA. (2017). Ley Federal de Derechos, Disposiciones aplicables en materia de aguas nacionales. Comisión Nacional de Agua, México.

Delgadillo A., González C., Prieto F., Villagómez J. y Acevedo O. (2011). Fitorremediación: una alternativa para eliminar la contaminación. Trop Subtrop Agroecosyt. 14 (2), 597-612.

Hernández M. (2009). Estudio de la Acumulación de Plomo y Cadmio por Asphodelus fistulosus L. y Brassica Juncea L. para fitorremediar jales. Tesis de Maestría, División de Ciencias Biológicas y de la Salud. Universidad Autónoma Metropolitana de Iztapalapa. Distrito Federal, México, 100 pp.

Kersten G., Majestic B. y Quigley, M. (2017). Phytoremediation of cadmium and lead-polluted watersheds. Ecotoxicol Environ Saf. 137, 225-232. DOI:10.1016/j.ecoenv. 2016.12.001.

Lasat M., Pence N., Garvin D., Ebbs S. y Kochian L. (2000). Molecular Physiology of zinc trasnport in the zinc hyperaccumulator Thlaspi caerulescens. J Exp Bot. 51 (342):71-9. DOI:10.1093/jexbot/51.342.71.

Luna Martínez F.J. (2011). Fitorremediación de suelos contaminados con metales pesados. Universidad 
Autónoma Antonio Narro. Tesis de Licenciatura, División de Carreras Agronómicas. Unidad Laguna. Universidad Autónoma Agraria Antonio Narro. Torreón, Coahuila, México, 81 pp.

Lyubenova L., Pongrac P., Vogel-Mikuš K., Mezek G.K., Vavpetič P., Grlj N., Regvar M., Pelicon P., y Schröder, P. (2013). The fate of arsenic, cadmium and lead in Typha latifolia: a case study on the applicability of micro-PIXE in plant ionomics. J Hazard Mater. 248, 371-378. DOI: /10.1016/j.jhazmat.2013.01.023.

Mench M., Schwitzguebel J.P., Schroeder P., Bert V., Gawronski S. y Gupta S. (2009). Assessment of successful experiments and limitation of phytotecnologies: contamination uptake, detoxification and sequestration, and consequences for food safety. Environ Sci Pollut Res. 16 (7), 876-900.

DOI: $10.1007 / \mathrm{s} 11356-009-0252-z$.

Moreno López M.V., González Pico L.F., Benavides Montoya A., Rubio Gómez A.D., y Miranda Navarro S.V. (2011). Cuantificación de Arsénico en Aguas Termales de Importancia Recreacional en el Estado de Chihuahua. Repositorio CIMAV, Chihuahua, México.

Núñez G., Alarcón M., Melgoza A., Rodríguez F. y Royo M. (2007). Evaluación de tres especies nativas del desierto Chihuahuense para uso en fitorremediación. Terra Latinoamericana, 25 (1), 35-41.

Olmos-Márquez M., Herrera-Alarcón M. y DomínguezMartín I. (2012). Performance of Eleocharis Macrostachya and its importance for arsenic retention in constructed wetlands. Environ Sci Pollut Res Int. 19 (3), 763-771. DOI: 10.1007/s11356-011-0598-x.

Ortiz H., Trejo R., Valdez R., Arreola J., Flores A. y López B. (2009). Fitoextracción de plomo y cadmio en suelos contaminados usando quelite (Amaranthus hybridus L.) y micorrizas. Rev. Chapingo Ser Hortic. 15(2), 161-168.

RECNAT. (2000). NORMA Oficial Mexicana NOM021-RECNAT-2000, Que establece las especificaciones de fertilidad, salinidad y clasificación de suelos. Estudios, muestreo y análisis. Secretaría de Medio Ambiente y Recursos Naturales. Diario Oficial de la Federación. 31 de Diciembre de 2002.

SEMARNAT. (1996). NORMA Oficial Mexicana NOM002-ECOL-1996, Que establece los límites máximos permisibles de contaminantes en las descargas de aguas residuales a los sistemas de alcantarillado urbano o municipal. Secretaría de Medio Ambiente y Recursos Naturales. Diario Oficial de la Federación. 3 de Junio de 1998.

Sheoran V., Sheoran, A.S., y Poonia P. (2016). Factors affecting phytoextraction: a review. Pedosphere. 26(2), 148-166. DOI:10.1016/S1002-0160(15)60032-7.

Tangahu B.V., Abdullah S., Rozaimah S., Basri H., Idris M., Anuar N., y Mukhlisin M. (2011). A review on heavy metals $(\mathrm{As}, \mathrm{Pb}$, and $\mathrm{Hg}$ ) uptake by plants through phytoremediation. Int J Chem Eng. 1-31. DOI: 10.1155/2011/939161.

Valles-Aragón M.C. y Alarcón-Herrera M.T. (2014). Retención de arsénico en humedales construidos con Eleocharis macrostachya y Schoenoplectus americanus. Rev Int Contam Ambie. 30 (2), 143-148.

Vera A., Ramos K., Camargo E., Andrade C., Núñez M. y Delgado J. (2016). Fitorremediación de aguas residuales con alto contenido de plomo utilizando Typha dominguensis y Canna generalis. Rev Téc Ing Univ Zulia. 39(2), 88-95.

Verbruggen N., Hermans C., y Schat H. (2009). Mechanisms to cope with arsenic or cadmium excess in plants. Plant Biology, 12:364-372. DOI: 10.1016/j.pbi.2009.05.001.

Yadav K.K., Gupta N., Kumar A., Reece L.M., Singh N., Rezania S., y Khan S.A. (2018). Mechanistic understanding and holistic approach of phytoremediation: A review on application and future prospects. Ecol Eng. 120, 274-298.

DOI: $10.1016 /$ j.ecoleng.2018.05.039. 